ARTIGO DE REVISÃO

\title{
Avaliação da performance técnico-tática no tênis: uma revisão sistemática sobre os instrumentos de medida
}

\author{
Technical-tactical performance assessment in tennis: a systematic review of measurement instruments
}

Rodrigo Vieira Bulso ${ }^{1}$, Gabriel Henrique Treter Gonçalves ${ }^{2}$, Thiago José Leonardi ${ }^{1}$, Carlos Adelar Abaide Balbinotti ${ }^{1}$

${ }^{1}$ Universidade Federal do Rio Grande do Sul (UFRGS), Porto Alegre/RS, Brasil

2 Universidade do Estado de Santa Catarina (UDESC), Florianópolis/SC, Brasil

\section{HISTÓRICO DO ARTIGO}

Recebido: 16 abril 2020

Revisado: 25 julho 2020

Aprovado: 27 julho 2020

\section{PALAVRAS-CHAVE:}

Estudos de Validação; Desempenho Atlético; Esportes; Tênis; Revisão sistemática.

\section{KEYWORDS:}

Validation Studies;

Athletic Performance; Sports;

Tennis; Systematic Review.

\section{RESUMO}

OBJETIVO: O estudo teve como objetivo revisar a literatura a fim de identificar instrumentos de avaliação da performance técnico-tática no tênis.

MÉTODOS: Foi realizado um estudo de revisão sistemática. A busca por artigos foi realizada nas bases de dados SCOPUS e WEB OF SCIENCE, utilizando-se os termos tennis AND performance AND assessment. Os critérios de inclusão estabelecidos para este estudo foram: (1) artigos de validação de instrumentos; (2) que tratassem de pelo menos um aspecto técnico ou tático; (3) que fossem específicos do tênis. Dos 422 artigos encontrados, sete foram selecionados para esta revisão.

RESULTADOS: Os instrumentos selecionados avaliavam em sua maioria os aspectos técnico-táticos do tênis de forma integrada, contemplando desde o público infantil até o público adulto de alto rendimento. Foi observado um maior número de instrumentos de avaliação no contexto do treino em relação ao contexto da competição. Todos os estudos apresentaram ao menos uma evidência de validade, e apenas dois estudos não apresentaram evidências de confiabilidade.

CONCLUSÃO: Esta revisão pode auxiliar treinadores e pesquisadores da área a escolher o instrumento de avaliação mais adequado de acordo com o contexto e os objetivos almejados.

\section{ABSTRACT}

OBJECTIVE: The study aimed to review the literature to identify instruments for assessing technical-tactical performance in tennis.

METHODS: A systematic review study was carried out. The search for articles was carried out in the SCOPUS and WEB OF SCIENCE databases, using the terms tennis AND performance AND assessment. The inclusion criteria established for this study were: (1) instrument validation articles; (2) dealing with at least one technical or tactical aspect; (3) that were specific to tennis. From a range of 422 articles found, we selected seven for this review.

RESULTS: The selected instruments evaluated mostly the technical-tactical aspects of tennis in an integrated way, covering from the child community to the high performance adult community. We observed a greater number of assessment instruments applied to the context of training in relation to the competition context. All studies presented at least one evidence of validity, and only two studies did not show evidence of reliability. CONCLUSION: This review can help coaches and researchers to choose the most appropriate assessment tool according to the context and to their targeted goals. 


\section{INTRODUÇÃO}

A avaliação da performance técnico-tática dos tenistas pode ser considerada um dos fatores mais importantes para o sucesso na modalidade, uma vez que possibilita aos treinadores (e aos próprios jogadores) o reconhecimento das principais qualidades e limitações dos atletas, servindo inclusive para balizar e aprimorar todo o processo de treino (CUI et al., 2017; 2018). Dentre as formas de avaliação da performance no tênis, para além da análise dos resultados e dados das partidas - número de bolas vencedoras, erros não-forçados, quantidades de pontos ganhos e perdidos, entre outros - e dos questionários, destacam-se os instrumentos de observação (TORRES-LUQUE et al., 2018). Os instrumentos de observação e análise de jogo tiveram início nos anos 1930, mas adquiriram maior ênfase na literatura, em especial nos esportes coletivos, a partir dos anos de 1980 (GARGANTA, 2001). Em síntese, eles permitem observar as ocorrências das ações durante a realização do jogo, em respeito à ecologia do jogo (DAVIS; BURTON, 1991; ARAÚJO et al., 2010).

A escolha de um instrumento adequado constitui um passo importante para se atestar a fidelidade dos resultados das pesquisas (SOUZA; ALEXANDRE; GUIRARDELLO, 2017). Na literatura especializada, diversos são os estudos que buscam validar instrumentos de avaliação da performance técnica e/ou tática no esporte (GONÇALVES et al., 2019). Apesar de grande parte destes instrumentos terem sido elaborados para os esportes coletivos, tanto para serem aplicados a diferentes modalidades - como o Team Sport Assessment Procedure (TSAP; GRÉHAIGNE; GODBOUT; BOUTHIER, 1997) e o Game Performance Assessment Instrument (GPAI; OSLIN; MITCHELL; GRIFFIN, 1998) - ou para modalidades específicas - como o Sistema de Avaliação Tática no Futebol (FUT-SAT; COSTA et al., 2011) -, são encontrados na literatura importantes instrumentos de avaliação da performance tática e/ou técnica específicos para o tênis (KOLMAN et al., 2018). No entanto, pouco se sabe sobre as características e a aplicabilidade dos mesmos.

Diante desse contexto, faz-se necessário investigar os instrumentos de avaliação específicos para o tênis. Assim, o presente estudo tem como objetivo revisar a literatura a fim de identificar instrumentos de avaliação da performance técnico-tática no tênis. Nesse sentido, foram considerados os seguintes aspectos: a) data de publicação; b) público-alvo; c) conteúdos avaliados; d) contexto de aplicação; e) protocolo; f) evidências de validade; g) evidências de confiabilidade.

\section{MÉTODOS}

Foi realizado um estudo de revisão sistemática (SAMPAIO; MANCINI, 2006) cujas etapas de seleção dos artigos foram realizadas pelo primeiro autor, sob supervisão do terceiro autor. Os critérios de inclusão estabelecidos para este estudo foram: (1) artigos de validação de instrumentos; (2) que tratassem de pelo menos um aspecto técnico ou tático; (3) que fossem específicos do tênis. A busca foi realizada nas bases de dados SCOPUS e Web of Science (WoS), utilizando-se os termos tennis AND performance AND assessment. Foram estabelecidos como critérios de exclusão: artigos de revisão, livros e capítulos de livros, resumos, monografias, dissertações e teses. Cabe salientar que não houve restrição de data da publicação dos artigos durante o processo de busca, e a última consulta foi realizada no dia 05 de dezembro de 2019.

Inicialmente, foram encontrados 422 artigos (SCOPUS = 249; WEB OF SCIENCE $=173$ ). Destes, 86 eram artigos duplicados, 319 não eram estudos de validação de instrumento, 6 não tratavam de aspectos técnicos ou táticos e 4 não eram específicos do tênis. Assim, foram incluídos para a presente revisão sistemática 7 artigos (SCOPUS = 1; SCOPUS/WEB OF SCIENCE = 6), publicados entre os anos de 2000 e 2019 (Figura 1).

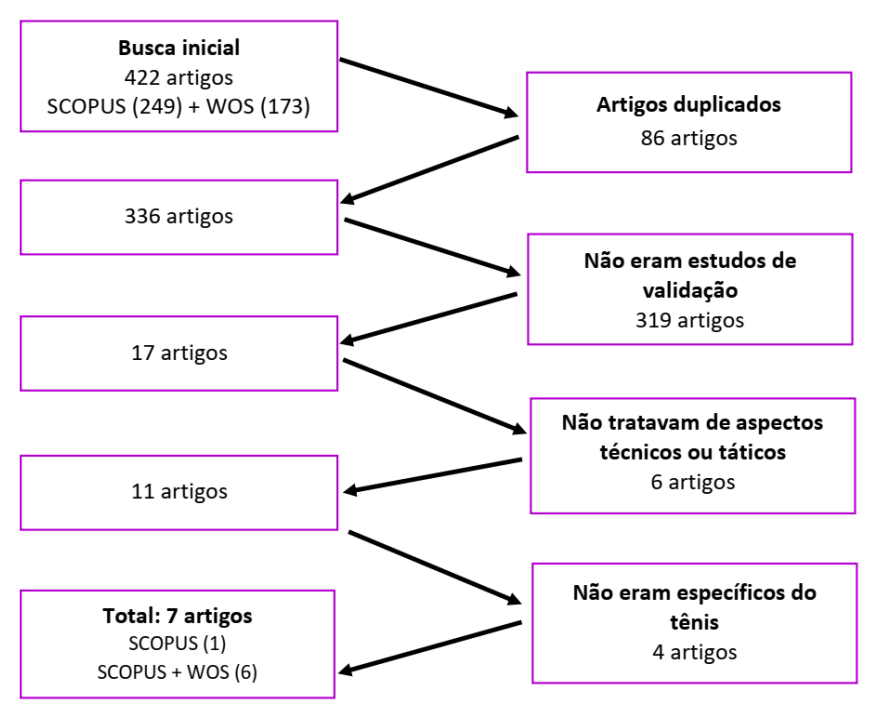

Figura 1. Organograma das etapas do processo de seleção dos artigos.

Os estudos foram analisados levando-se em consideração os seguintes critérios: a) Data de publicação (Estudos recentes = de 2016 a 2020; Estudos antigos = de 2000 à 2015); b) Público-alvo (Crianças = de 6 até 12 anos de idade; Adolescentes = de 13 a 17 anos; Adultos = a partir de 18 anos; Todas as idades); c) Conteúdos avaliados (Tático; Técnico; Técnico-Tático); d) Contexto de aplicação (Competição; Treino); e) Protocolo (Com observação; Sem observação); f) Evidências de validade (Uma; Duas; Três ou mais); g) Evidências de confiabilidade (Sem evidências; Uma; Duas).

\section{RESULTADOS}

No Quadro 1 são apresentados os sete artigos selecionados na presente revisão sistemática, contendo informações referentes ao periódico, autores, data de publicação, nome do instrumento, conteúdo(s) avaliado(s), público-alvo, contexto de aplicação, protocolo, evidências de validade e de confiabilidade do instrumento.

\section{Data de publicação}

Os artigos mais antigos que atenderam aos critérios de inclusão da presente revisão foram publicados nos anos de 2000 (REES; HARDY; INGLEDEW, 2000) e 2004 (VERGAUWEN; MADOU; BEHETS, 2004). Já, a maior parte dos instrumentos (cinco) foram publicados nos últimos 5 anos (VAVERKA; CERNOSEK, 2016; KOLMAN et al., 2017; KRAUSE et al., 2017; SLOSAR et al., 2018; TORRES-LUQUE et al., 2018). A constatação de que a maior parte dos instrumentos validados foram publicados 
Quadro 1. Estudos de validação de instrumentos de avaliação da performance técnico-tático no tênis.

\begin{tabular}{|c|c|c|c|c|c|c|}
\hline Autores (ano) & Nome do instrumento & $\begin{array}{l}\text { Conteúdo(s) } \\
\text { avaliado(s) }\end{array}$ & $\begin{array}{l}\text { Público } \\
\text { Alvo }\end{array}$ & $\begin{array}{l}\text { Contexto de } \\
\text { aplicação }\end{array}$ & $\begin{array}{l}\text { Evidências de } \\
\text { Validade/ } \\
\text { Confiabilidade }\end{array}$ & Protocolo \\
\hline $\begin{array}{l}\text { Rees, Hardy e } \\
\text { Ingledew (2000) }\end{array}$ & $\begin{array}{l}\text { Measurement Instrument for Tennis Performance } \\
\text { (MITP) }\end{array}$ & Tático & Adultos & Competição & Quatro/Sem & Sem observação \\
\hline $\begin{array}{l}\text { Vergauwen, Madou e } \\
\text { Behets (2004) }\end{array}$ & Foreground & Técnico-tático & Adolescentes & Treino & Uma/Duas & Com observação \\
\hline $\begin{array}{l}\text { Vaverka; Cernosek } \\
\text { (2016) }\end{array}$ & $\begin{array}{l}\text { Coefficient of Serve Speed } \\
\text { (CSS) }\end{array}$ & Técnico & Adultos & Treino & Uma/Sem & Sem observação \\
\hline Kolman et al. (2017) & $\begin{array}{l}\text { Dutch Technical-Tactical Tennis Test } \\
\text { (D4T) }\end{array}$ & Técnico-tático & Adolescentes & Treino & Duas/Uma & Com observação \\
\hline Krause et al. (2017) & $\begin{array}{l}\text { Representative Practice Assessment Tool } \\
\text { (RPAT) }\end{array}$ & Técnico-tático & Todas as idades & Treino & Uma/Uma & Com observação \\
\hline $\begin{array}{l}\text { Torres-Luque et al. } \\
\text { (2018) }\end{array}$ & $\begin{array}{l}\text { Observation instrument for singles tennis } \\
\text { (OIST) }\end{array}$ & Técnico-tático & Todas as idades & Competição & Uma/Duas & Com observação \\
\hline
\end{tabular}

em estudos recentes pode indicar um maior interesse dos pesquisadores acerca deste tema, em comparação aos anos anteriores. Este pode ser considerado um importante passo para a modalidade, uma vez que a utilização de tais ferramentas pode contribuir para qualificar o processo de treino e de avaliação da aprendizagem (CUI et al., 2018).

\section{Público-alvo}

Os instrumentos selecionados para esta revisão contemplam diferentes públicos-alvo. Ao passo que um instrumento foi elaborado especificamente para crianças de 6 a 12 anos (TRSC), dois foram validados para o público adolescente (Foreground e D4T) e dois para o público adulto (MITP e CSS). Nesse sentido, apesar de ter sido elaborado a partir da análise de vídeo de jogos profissionais masculinos, o Observation Instrument for Singles Tennis (OIST) foi proposto para ser utilizado com jogadores de todas as idades e níveis. Da mesma forma, o RPAT pode ser utilizado pelos treinadores para avaliar as tarefas de treino dos tenistas, independentemente do nível de jogo. O fato desses instrumentos contemplarem diferentes faixas etárias e níveis de jogo parecem evidenciar: a) a relevância deste tema na academia; b) a importância da avaliação da aprendizagem técnico-tática dos tenistas, desde a fase de iniciação esportiva até $o$ alto rendimento adulto.

\section{Conteúdos avaliados}

Dos sete instrumentos de avaliação da performance incluídos nesta revisão, apenas um busca avaliar os aspectos táticos de forma isolada - implementação de um plano de jogo e táticas eficazes (REES; HARDY; INGLEDEW, 2000) -, dois buscam avaliar apenas os aspectos técnicos - execução do forehand, backhand e saque (SLOSAR et al., 2018); coeficiente de velocidade do saque em relação à estatura (VAVERKA; CERNOSEK, 2016) -, e três buscam avaliar os aspectos técnico-táticos de forma integrada - índice de acertos, velocidade e precisão do forehand (VERGAUWEN; MADOU; BEHETS, 2004); velocidade e precisão do forehand e backhand, e porcentagem de erros (KOLMAN et al., 2017); sequência de golpes, tipos de técnicas e táticas utilizadas, posicionamento no momento da rebatida, entre outros (TORRES-LUQUE et al, 2018). Ainda, o RPAT (KRAUSE et al.,
2017) foi elaborado com o intuito de avaliar o objetivo e a relevância das tarefas de treino, do ponto de vista técnico-tático.

A predominância de instrumentos de medida relacionados aos conteúdos técnico-táticos de forma integrada parece ser um bom indicativo. A constante interação entre as habilidades técnicas e táticas é um fator preponderante na performance dos tenistas durante o jogo, e, portanto, analisar separadamente as habilidades técnicas ou táticas são fatores limitadores importantes nos estudos relacionados ao tênis (KOLMAN et al., 2018). A maior quantidade de instrumentos que buscam avaliar os aspectos técnicos de forma isolada, em comparação aos aspectos táticos, também foi verificada em estudo de revisão anterior (GONÇALVES et al., 2020).

\section{Contexto de aplicação}

Dos sete instrumentos selecionados para a presente revisão, dois foram propostos para serem utilizados no contexto da competição: um referente à observação de jogos (OIST), e um para ser respondido pelos próprios jogadores após o término das partidas (MITP). Os outros cinco instrumentos podem ser utilizados no contexto do treino, como o CSS e o RPAT. Dois destes cinco instrumentos foram propostos para serem aplicados em quadra oficial, a partir de lançamento de bolas realizados pelo treinador (Foreground) ou por uma máquina de lançar bolas (D4T); e um em uma quadra de tênis com dimensões reduzidas $-13 \mathrm{~m} \times 6 \mathrm{~m}$; altura da rede de $80 \mathrm{~cm}-$ (TRSC), a partir de lançamentos de bolas realizado por um professor treinado.

Com a crescente importância do jogo no processo de ensino e treino do tênis (UNIERZYSKI; CRESPO, 2007), há de se ressaltar a relevância dos instrumentos de avaliação da performance no contexto competitivo. O OIST, por exemplo, contempla a avaliação do desempenho nos jogos. Com intuito de padronizar os testes e avaliar conteúdos específicos, alguns procedimentos visam recriar em quadra situações próximas às encontradas nas partidas - através do lançamento de bolas neutras, ofensivas e/ ou defensivas (VERGAUWEN; MADOU; BEHETS, 2004; KOLMAN et al., 2017). Ainda, o fato de haver um instrumento que avalia a performance de tenistas até 12 anos em ambiente reduzido se mostra de acordo com o que propõe a literatura especializada acerca dos processos de treino de tenistas dessa faixa de idade (FARROW; REID, 2010; BUSZARD et al., 2014). 


\section{Protocolo}

Cinco dos sete instrumentos selecionados para este estudo dependem da observação em vídeo realizada pelos avaliadores. Em dois destes cinco instrumentos (Foreground e D4T), os avaliadores são responsáveis por indicar o ponto de aterrisagem da bola rebatida pelo tenista e classificar esta área de acordo com os critérios estabelecidos nos testes. No OIST são observadas 23 variáveis -10 contextuais, 8 de resultado e 5 de jogo -, contemplando aspectos técnico-táticos como a sequência de golpes no ponto, os tipos de golpe utilizados, o local em que o golpe foi realizado, o ponto de aterrisagem da bola rebatida e a efetividade dos golpes. No TRSC é realizada a observação e classificação das técnicas de saque, forehand e backhand dos jovens, levando-se em consideração cada uma das cinco fases preestabelecidas: preparação; aceleração; ponto de contato; follow trough; e cadeia cinética. No RPAT as tarefas de treino são observadas e classificadas, levando-se em consideração sete categorias estabelecidas pelos especialistas (objetivo da tarefa, maneira com que a primeira bola é lançada, contribuição para a tomada de decisão, entre outros). Ainda, dois destes não são instrumentos de observação, uma vez que o MITP consiste em um questionário a ser respondido pelos jogadores, e a velocidade do saque no CSS é medida através de um radar.

\section{Evidências de validade}

Todos os artigos publicados selecionados para esta revisão apresentaram ao menos uma evidência de validade do instrumento. No CSS foi testada a validade estatística. No Foreground, RPAT e OIST foram apresentadas evidências da validade de conteúdo. No TRSC foram testadas, além da validade de conteúdo, a validade de face e a validade convergente. Kolman et al. (2017) apresentaram evidências das validades concorrente e discriminante do D4T. Ainda, Rees, Hardy e Ingledew (2000) testaram em seu estudo a validade estrutural, fatorial, convergente e discriminante do MITP.

Tendo em vista que a validade e a confiabilidade dos instrumentos de avaliação são premissas da metodologia científica (CHACÓN-MOSCOSO et al., 2018), o fato de que todos os artigos selecionados para este estudo apresentaram ao menos uma evidência de validade é de extrema relevância. No entanto, cabe destacar que nem todos os estudos apresentaram de forma clara e objetiva os procedimentos utilizados no processo de validação dos instrumentos.

\section{Evidências de confiabilidade}

A confiabilidade dos instrumentos foi testada e descrita detalhadamente na maior parte dos artigos, que apresentaram de uma a duas evidências relacionadas a este aspecto - confiabilidade interobservador e/ou intraobservador. Ao total, cinco estudos apresentaram evidências de confiabilidade do instrumento validado. Destes, três estudos testaram a confiabilidade intraobservador e interobservador (Foreground, TRSC e OIST), e dois estudos testaram apenas a confiabilidade interobservador (D4T e RPAT). Ainda, dois estudos não apresentaram evidências de confiabilidade do instrumento proposto. Destes, um se tratava de um questionário a ser respondido pelos próprios jogadores (MITP) e outro estava relacionado à medição da velocidade do saque (CSS).
No TRSC, a confiabilidade intraobservador e interobservador foram medidas através do Índice de Correlação Intraclasse (ICl; sendo ICIINTRA > 0,88; ICIINTER $\geq 0,87$ ). No Foreground também foram utilizados os valores de ICI (ICIINTRA = 0,99; ICIINTER $=0,99$ ) para evidenciar a confiabilidade do instrumento. Cabe destacar que valores de $\mathrm{ICl}$ superiores a 0,75 são considerados excelentes. A confiabilidade intraobservador e interobservador do OIST foi medida através do coeficiente Kappa de Cohen (KINTRA > 0,9; KINTER > 0,8), mesmo índice utilizado para medir a confiabilidade interobservador do D4T (KINTER $=0,98)$. Valores superiores a 0,80 para o Kappa também são considerados excelentes. Ainda, a confiabilidade interobservador do RPAT foi avaliada através da medição dos desvios-padrão das classificações dos itens $(D P=0,77)$, sendo valores inferiores a 1 considerados bons para este tipo de cálculo.

\section{Principais contribuições}

Ao analisar individualmente cada um dos instrumentos abordados, é possível apontar suas principais contribuições para o processo de avaliação da performance técnico-tática, bem como algumas limitações. Dentre estes, o único instrumento proposto para avaliar a performance do tenista durante a partida é o OIST, que leva em consideração diferentes parâmetros relacionados ao contexto da competição e à performance do jogador, além de poder ser utilizado para os mais diferentes níveis e faixas etárias. O MITP pode ser utilizado de forma conjunta ou complementar ao OIST, uma vez que contempla aspectos relacionados à implementação do plano de jogo e táticas eficazes, sendo respondido pelos atletas logo após as partidas.

Ao se pensar a aplicabilidade prática dos instrumentos no contexto do treino, destacam-se o Foreground - no caso do público infantil - e o TRSC - no caso do público adolescente -, pois ambos os testes podem ser realizados a partir de lançamentos realizados pelo próprio treinador. Essa caraterística torna mais fácil a reprodução dos testes nas aulas e treinos, apesar de poder apresentar maior variação na velocidade, altura e ponto de aterrisagem - fator que poderia influenciar na performance técnica (no caso TRSC) ou técnico-tática dos jogadores (no caso do Foreground).

Estes testes foram elaborados para jogadores de nível iniciante e/ou intermediário. Em contraponto, para a aplicação do D4T é necessária a utilização uma máquina de lançar bolas, o que pode dificultar sua aplicação prática, ao passo que aumenta o nível de precisão dos lançamentos. Cabe ressaltar que o D4T é um teste já utilizado pela Federação Alemã de Tênis com tenistas adolescentes, configurando-se um teste mais complexo e, portanto, indicado para jogadores com nível de jogo mais avançado.

O CSS, por sua vez, é um instrumento bastante específico, pois avalia, a partir da medição da velocidade do saque, o coeficiente da velocidade do saque dos jogadores, comparando-os com os de tenistas profissionais mais bem ranqueados - e de mesma estatura. Seus resultados podem indicar a necessidade de treinamentos técnicos ou físicos específicos para a melhora do rendimento nesse golpe. Ainda, diferentemente dos outros instrumentos, o RPAT foi desenvolvido para que os treinadores possam avaliar suas tarefas de treino quanto ao seu objetivo e relevância para o desempenho competitivo dos jogadores, auxiliando-os de forma direta na melhoria do planejamento dos treinos. 


\section{CONCLUSÃO}

Este estudo analisou instrumentos de avaliação da performance técnico-tática específicos para o tênis, de acordo com sua data de publicação dos artigos, com os conteúdos avaliados, o público-alvo, os contextos de aplicação, os protocolos e as evidências de validade e confiabilidade apresentadas. De modo geral, os instrumentos foram validados nos últimos 5 anos. Em suma, buscavam avaliar a performance técnico-tática de forma integrada, em diferentes faixas etárias (crianças, adolescentes e adultos) e no contexto do treino. A validade e a confiabilidade foram apresentadas na maior parte dos estudos, indicando certa robustez aos procedimentos propostos. É possível concluir que, de fato, estes são instrumentos que apresentam evidências de validade para a utilização no contexto do tênis. No entanto, reitera-se que o processo de validação dos instrumentos de avaliação da performance técnico-tática deve ser contínuo e sistemático, no sentido de que estes instrumentos devem seguir apresentando evidências de validade por diferentes vieses e demonstrando que a avaliação não sofre uma eventual influência temporal ou de evolução do jogo/jogadores.

Ao analisar as principais características de cada instrumento, esta revisão pode auxiliar treinadores e pesquisadores da área a escolher o instrumento de avaliação da performance técnica e/ ou tática mais adequado de acordo com o contexto e os objetivos almejados. Além disso, a apresentação dos manuais de aplicação dos instrumentos, verificada nos artigos, é de grande valia em termos práticos. O presente estudo pode contribuir, mesmo que indiretamente, com a melhora dos processos avaliativos da modalidade, e, por conseguinte, dos processos de treino.

No entanto, é preciso tecer algumas considerações quanto às limitações deste estudo e suas perspectivas futuras. A busca pelos artigos em duas bases de dados e a utilização de palavras-chave bastante específicas não exclui a possibilidade de existirem outros instrumentos de avaliação da performance técnico-tática no tênis. A opção por incluir apenas instrumentos específicos do tênis também pode ser considerado um fator limitante, uma vez que instrumentos gerais podem apresentar evidências de validade para uma modalidade específica. Assim, sugere-se a realização de novos estudos que busquem analisar de forma mais abrangente os instrumentos de avaliação da performance técnico-tático que contemplem o tênis.

\section{REFERÊNCIAS}

ARAÚJO, D.; FONSECA, C.; DAVIDS, K.; GARGANTA, J.; VOLOSSOVITCH, A.; BRANDÃO, R.; KREBS, R. The role of ecological constraints on expertise development. Talent Development \& Excellence, Granada, v. 2, n. 2, p. 16579, 2010.

BUSZARD, T.; FARROW, D.; REID, M.; MASTERS, R. S. W. Modifying equipment in early skill development: a tennis perspective. Research Quarterly for Exercise And Sport, Cardiff, v. 85, n. 2, p. 218-25, 2014.

CHACÓN-MOSCOSO, S.; SANDUVETE-CHAVES, S.; ANGUERA, M. T.; LOSADA, L.; PORTELL, M.; LOZANO-LOZANO, J. A. Preliminary checklist for reporting observational studies in sports areas: content validity. Frontiers in Psychology, Lausanne, v. 9, p. 1-2, 2018.

COSTA, I. T.; GARGANTA, J.; GRECO, P.; MESQUITA, I.; MAIA, J. Sistema de avaliação táctica no futebol (FUT-SAT): desenvolvimento e validação preliminar. Motricidade, Ribeiro de Pena, v. 1, n. 7, p. 66-84, 2011.

CUI, Y.; GÓMEZ, M. A.; GONÇALVES, B.; LIU, H.; SAMPAIO, J. Effects of experience and relative quality in tennis match performance during four Grand Slams. International Journal Of Performance Analysis in Sport, Cardiff, v. 17, n. 5, p. 783-801, 2017.

CUI, Y.; GÓMEZ, M. A.; GONÇALVES, B.; SAMPAIO, J. Performance profiles of professional female tennis players in grand slams. Plos One, San Francisco, v. 13, n. 7, p. 1-18, 2018.

DAVIS, W. E.; BURTON, A. W. Ecological task analysis: translating movement behavior theory into practice. Adapted Physical Activity Quarterly, Champaign, v. 8, p. 154-77, 1991.

FARROW, D.; REID, M. The effect of equipment scaling on the skill acquisition of beginning tennis players. Journal of Sports Sciences, London, v. 28, n. 7, p. 723-32, 2010.

GARGANTA, J. Análise da performance nos jogos desportivos: revisão acerca da análise do jogo. Revista Portuguesa de Ciências do Desporto, Porto, v. 1, n. 1, p. 57-64, 2001

GONÇALVES, G. H. T.; BALBINOTTI, M. A. A.; GINCIENE, G.; CARDOSO, M. F. S.; KLERING, R. T.; BALBINOTTI, C. A. A. Desenvolvimento técnico-tático: evidências de validade de escalas de medida de conteúdos pedagógicos no esporte. Journal of Physical Education, Maringá, v. 30, p. 3060, 2019.

GONÇALVES, G. H. T.; BALBINOTTI, M. A. A.; BULSO, R. V.; KLERING, R. T.; BALBINOTTI, C. A. A. Conteúdos pedagógicos do esporte: uma revisão integrativa sobre instrumentos de medida. Saúde e Desenvolvimento Humano, Canoas, v. 8, p. 141-52, 2020.

GRÉHAIGNE, J. F.; GODBOUT, P.; BOUTHIER, D. Performance assessment in team sports. Journal of Teaching in Physical Education, Hanover, v. 16, n. 4, p. 500-16, 1997.

KOLMAN, N.; HUIJGEN, B. C. H.; KRAMER, T.; ELFERINK-GEMSER, M. T.; VISSCHER, C. Technical the dutch technical-tactical tennis test (d4t) for talent identification and development: psychometric characteristics. Journal of Human Kinetics, Katowice, v. 55, n. 1, p. 127-38, 2017.

KOLMAN, N.; KRAMER, T.; ELFERINK-GEMSER, M. T.; HUIJGEN, B. C. H.; VISSCHER, C. Technical and tactical skills related to performance levels in tennis: a systematic review. Journal of Sports Sciences, London, v. 37, n. 1, p. 108-21, 2018.

KRAUSE, L.; FARROW, D.; REID, M.; BUSZARD, T.; PINDER, R. Helping coaches apply the principles of representative learning design: validation of a tennis specific practice assessment tool. Journal of Sports Sciences, Katowice, v. 36, n. 11, p. 1277-86, 2017.

OSLIN, J.; MITCHELL, S.; GRIFFIN, L. The Game Performance Assessment Instrument (GPAI): development and preliminary validation. Journal of Teaching in Physical Education, London, v. 17, n. 2, p. 231-43, 1998.

REES, T.; HARDY, L.; INGLEDEW, D. K. Performance assessment in sport: Formulation, justification, and confirmatory factor analysis of a measurement instrument for tennis performance. Journal of Applied Sport Psychology, Indianapolis, v. 12, n. 2, p. 203-18, 2000.

SAMPAIO, R. F.; MANCINI, M. C. Estudos de revisão sistemática: um guia para síntese criteriosa da evidência científica. Revista Brasileira de Fisioterapia, São Carlos, v. 1, n. 11, p. 83-89, 2007.

SLOSAR, L.; SIMUNIC, B.; PISOT, R.; MARUSIC, U. Validation of a tennis rating score to evaluate the technical level of children tennis players. Journal of Sports Sciences, London, v. 37, n. 1, p. 100-7, 2018.

SouzA, A. C.; ALEXANDRE. N. M. C.; GuiRARdello, E. B. Propriedades psicométricas na avaliação de instrumentos: avaliação da confiabilidade e da validade. Epidemiologia e Serviços de Saúde, Brasília, v. 26, n. 3, p. 64959, 2017.

TORRES-LUQUE, G.; FERNÁNDEZ-GARCIA, A. I.; CABELLO-MANRIQUE, D.; GIMÉNEZ-EGIDO, J. M.; ORTEGA-TORO, E. Design and validation of an observational instrument for the technical-tactical actions in singles tennis. Frontiers in Psychology, Lausanne, v. 9, p. 1-10, 2018.

UNIERZYSKI, P.; CRESPO, M. Review of modern teaching methods for tennis (Análisis de los métodos actuales de enseñanza del tenis). Revista Internacional de Ciencias del Deporte, San Javier, v. 3, n. 7, p. 1-10, 2007. 
VAVERKA, F; CERNOSEK, M. Quantitative assessment of the serve speed in tennis. Sports Biomechanics, London, v. 15, n. 1, p. 48-60, 2016.

VERGAUWEN, L.; MADOU, B.; BEHETS, D. Authentic evaluation of forehand groundstrokes in young low- to intermediate-level tennis Players. Medicine \& Science in Sports \& Exercise, Indianapolis, v. 36, n. 12, p. 2099-2106, 2004.

\section{CONFLITO DE INTERESSE}

Os autores do estudo declaram não haver conflito de interesses.

\section{FINANCIAMENTO}

Este estudo não teve apoio financeiro.

\section{ORCID E E-MAIL DOS AUTORES}

Rodrigo Vieira Bulso (Autor Correspondente) ORCID: 0000-0002-7555-3094.

E-mail: rodrigo.bulso@gmail.com

Gabriel Henrique Treter Gonçalves ORCID: 0000-0001-8048-7565.

E-mail: gabriel.goncalves@udesc.br

Thiago José Leonardi

ORCID: 0000-0002-3843-2648.

E-mail: thiago.leonardi@ufrgs.br

Carlos Adelar Abaide Balbinotti

ORCID: 0000-0002-6358-1848.

E-mail: carlos.balbinotti@ufrgs.br 